\title{
Emotional Versus Neutral Expressions and Perceptions of Social Dominance and Submissiveness
}

\author{
Shlomo Hareli and Noga Shomrat \\ University of Haifa
}

\author{
Ursula Hess \\ University of Quebec at Montreal
}

\begin{abstract}
Emotional expressions influence social judgments of personality traits. The goal of the present research was to show that it is of interest to assess the impact of neutral expressions in this context. In 2 studies using different methodologies, the authors found that participants perceived men who expressed neutral and angry emotions as higher in dominance when compared with men expressing sadness or shame. Study 1 showed that this is also true for men expressing happiness. In contrast, women expressing either anger or happiness were perceived as higher in dominance than were women showing a neutral expression who were rated as less dominant. However, sadness expressions by both men and women clearly decreased the extent to which they were perceived as dominant, and a trend in this direction emerged for shame expressions by men in Study 2. Thus, neutral expressions seem to be perceived as a sign of dominance in men but not in women. The present findings extend our understanding of the way different emotional expressions affect perceived dominance and the signal function of neutral expressions-which in the past have often been ignored.
\end{abstract}

Keywords: social perception of emotions, emotional expression, social dominance, social submissiveness

Showing certain emotion expressions leads others to attribute specific traits to the individuals who express these emotions and, conversely, knowledge that a person has certain traits leads people to expect certain emotional reactions from them. One important set of traits in this context is related to social power.

Social power refers to the ability of an individual to provide or withhold valued resources or administer punishment (Anderson \& Berdahl, 2002). Two important correlates of social power that have been found to be associated with specific facial expressions are status (Tiedens, Ellsworth, \& Mesquita, 2000), which describes the power associated with the individual's role, and dominance (Hess, Adams, \& Kleck, 2005), which describes how assertive, forceful, and/or self-assured an individual is - these factors in turn impinge on a person's potential power (for a fuller discussion, see Anderson \& Berdahl, 2002; Keltner, Gruenfeld, \& Anderson, 2003).

In this context, Tiedens et al. (2000) found that participants believed that a high-status person would feel more anger when failing and more pride when succeeding compared with a person of lower status who is expected to feel more sadness/guilt versus appreciation in the respective situations. Conversely, observers perceive anger as a more appropriate reaction for a dominant person than for a submissive one (Hess et al., 2005).

Overall, these findings are consistent with the notion put forward by Keltner et al. (2003), that high levels of power are associated with the approach system, and the cognitions, emotions, and behaviors that are related to approach, whereas lower levels of

Shlomo Hareli and Noga Shomrat, Graduate School of Management, School of Management, University of Haifa; Ursula Hess, University of Quebec at Montreal.

Correspondence concerning this article should be addressed to Shlomo Hareli, School of Management, University of Haifa, Haifa, Israel 31905. E-mail: shareli@gsb.haifa.ac.il power are related to the inhibition system and the cognitions, emotions, and behaviors it connects to. Accordingly, emotions such as guilt, sadness, shame, embarrassment, and so forth that are related to the inhibition system are also linked to low power, whereas emotions such as pride, amusement, and happiness are associated with the approach system and thus with high power (Anderson \& Berdahl, 2002).

The connection between information about social power and expected emotional reactions also leads to the converse effects. That is, observers make status or dominance inferences based on the emotions that an individual expresses in a given situation. For example, if two individuals are known to have failed at a task and one of them shows anger whereas the other expresses guilt, the angry person will be perceived as higher in status than the guilty one (Tiedens, 2001; Tiedens et al., 2000; and see Aguinis, Simonsen, \& Pierce, 1998; Hess, Blairy \& Kleck, 2000; Knutson, 1996, for related findings regarding perceptions of dominance).

These findings suggest that observers hold a naïve theory that closely matches Keltner et al.'s (2003) model and associate high power (as well as status and social dominance) with approach-related emotions such as anger and pride, and low power/status/dominance with inhibition-related emotions such as sadness and fear. The effect of anger versus sadness, shame, guilt, and so forth as a signal of power is well established. However, this line of research has largely ignored neutral expressions and thereby limited a fuller understanding of the way discrete emotional expressions contribute to inferences of power. First, because most of the research tapped the impact of one emotion relative to another on perceptions of power (e.g., Hess et al., 2000; Tiedens, 2001), it remains as yet unclear whether the expression of approach-related emotions (e.g., anger) make the person who expresses the emotions appear more powerful or whether the expression of emotions associated with the inhibition system (e.g., guilt and sadness) make that indi- 
vidual appear less powerful or both. Research and discussions in the past seem to implicitly imply that any difference in perception of power between an individual who expressed approach-related emotions with an individual who expressed inhibition-related emotions is due to the two types of emotions pushing perceptions into the opposite direction. That is, approach-related emotions cause an increase in perceived power and inhibition-related emotion decrease perceived power (e.g., Keltner et al., 2003; Tiedens, 2001). To understand the actual contribution of each emotional expression to perception of power, it is necessary to compare the impact of expressions of emotions such as anger or sadness or guilt to the impact of an emotionally neutral expression.

There are in fact reasons to assume that the differential impact of approach versus inhibition-related emotions on perceptions of power are mostly driven by emotions associated with inhibition reducing the level of perceived power rather than approach-related emotions increasing it. One reason for this idea comes from the expectation that neutral expressions are powerful in their own right. As Watzlawick famously noted "one cannot not communicate" (cf. Wilder, 1978) and hence not reacting to a situation signals something about the person who does not react. More specifically, recently it has been argued that restrained emotion displays are suggestive of mastery of one's live and of competence in general (Warner \& Shields, 2007) - conversely, relaxedness, as far as this is reflected in one's voice, is associated with high status and dominance (Hall $\&$ Friedman, 1999). Similarly, showing any emotional reaction to an event may at times be seen as a sign of weakness (Kopelman, Rosette, \& Thompson, 2006). Taken together, this may suggest that reacting nonemotionally to a given event or situation by showing a neutral expression is likely to lead to an impression of an ability to handle the situation, which is generally associated in people's minds with social power and dominance (Tiedens, 2001). In fact, it may be the case that reacting nonemotionally is even more characteristic of a high power individual than reacting to an event emotionally by showing anger. That this may indeed be the case-at least for women - was shown by Lewis (2000) who found that female managers were perceived as most competent when retaining a neutral demeanor in the face of bad news.

Based on the above rationale, the goal of the present research was to explore how neutral expressions affect perception of power relative to approach and inhibition emotions. More specifically, we investigated whether (a) expressions of approach emotions increase perceptions of power, (b) expressions of inhibition emotions decrease perceptions of power, or (c) both is the case by comparing their impact with a neutral expression. A second goal was to assess the impressions entrained by the neutral expressions; a relatively neglected question in prior research. By using a neutral expression, this research will enable a closer understanding of the way different discrete emotions affect perceptions of power.

\section{Overview of the Present Research}

Two studies were conducted using the general paradigm employed in previous research, to test the specific contribution of approach and inhibition emotions as well as neutral expres- sions to perceptions of dominance. In Study 1, observers saw still photos of men and women showing different facial expressions and judged their social dominance. We included both men and women because prior research showed that observers are sensitive to a person's gender when making dominance judgments (Hess et al., 2005). For Study 2, we provided a specific social context in which the judgments were made. Specifically, observers watched a video in which a male employee who had failed at a task explains the reasons that brought about his failure. Several measures of dominance were used in order to increase the validity of our results. The two studies were conducted in different cultures varying in power distance, that is, the extent to which people in that culture are tolerant to power differences (Hofstede, 2001). In general, Canadians are more tolerant to power distance than Israelis, who rank very low on this measure (Aycan et al., 2000; Hofstede, 2001). Also, the Israeli sample is comprised of somewhat older people than the Canadian one. This may also impinge on perceptions related to power because age is known to be associated with power (Berger, Cohen, \& Zelditch, 1972; Mazur, 1985). Overall, the use of different methodologies, contexts, measures, cultures, and age groups sets the stage for potentially more generalizeable results.

Based on theoretical considerations mentioned earlier, we expected that anger and happiness will increase perceptions of dominance relative to inhibition-related expressions but not relative to neutral expressions. In contrast, we expected inhibition-related emotions (fear, sadness and shame) to decrease perceived power relative to both approach-related emotional expressions and neutral ones.

\section{Study 1}

\section{Method}

Participants. A total of 86 men and 122 women and two gender-unknown individuals with a mean age of 27 years $(S D=$ 8.7) participated in the study. Of these, 34 had completed high school, 98 had some level of university or vocational college education, and 73 had a university degree.

Stimulus material. Using the directed facial action task (DFA; Levenson, Ekman, \& Friesen, 1990), two men and two women (aged between 24 and 30 years) were instructed to pose facial expressions of happiness, sadness, fear, anger, and emotional neutrality. This procedure has been employed for the creation of standard sets of emotional facial expressions that are widely used by researchers in the field such as the Japanese and Caucasian facial expressions of emotion (JACFEE; Matsumoto \& Ekman, 1988) and the Montreal set of facial displays of emotion (MSFDE; Beaupré \& Hess, 2005).

The expressions were simultaneously filmed face on and in [3/4] profile from the left side. This resulted in a total of 4 (actors) $\times 5$ (four emotions + neutral) $=20$ stimuli for each angle; in the framework of the present study only ratings of face on stimuli will be considered. The stimuli were presented in a between-subjects design. Each participant saw a random combination of four stimuli (with the restriction that no actor was shown twice) and was asked to rate these on the dependent variables described below. The facial expressions were well recognized by a separate sample of judges who showed a high level of agreement in rating the emo- 
tions expressed in these photos as this is reflected in relative high interclass correlations (ICCs for anger, fear, happiness, neutrality, and sadness were $.80, .98, .99, .79$, and .99 , respectively; for more details, see Hess, Adams, \& Kleck, 2007).

Procedure. Participants were recruited in parks and public places in the Montreal urban area, as well as in classrooms at the University of Quebec at Montreal. Participants were given a clipboard with four pages. Each page showed a face stimulus and the rating scales. They rated each face on four 7-point scales ranging from -3 to +3 : assertive-insecure, forceful-placid, controlling-noncontrolling, and dominant-submissive. The subscales were combined into an overall dominance scale $(\alpha=.76)$. Following the task, participants completed a brief demographic data form.

Data analyses. Because each judge saw only a small random sample of the total stimuli (with the above mentioned restriction), the dependent measures were analyzed using a complete betweensubjects analyses. The intraclass correlation for the stimuli was -.10 , suggesting a low level of dependence and hence justifying this approach.

\section{Results}

For the dominance ratings no significant main effect nor significant interactions involving the sex of the rater emerged. This factor was therefore dropped from further analysis. A 5(Emotion) $\times 2$ (Expresser gender) analysis of variance revealed the predicted main effect of emotion expression, $F(4,404)=42.02$, $p<.001, \eta^{2}=.29$. Furthermore, a significant main effect of expresser sex, $F(1,404)=4.40, p=.037, \eta^{2}=.01$ emerged. As predicted these effects were qualified by a significant emotion expression $\times$ expresser sex interaction, $F(4,404)=3.11, p=$
$.015, \eta^{2}=.03$. We therefore conducted the a priori comparison $(p<.05)$ comparing dominance ratings for the four emotion expressions with the neutral expression, separately for male and female expressers. Men who showed anger or happiness were not perceived as more dominant than those who showed neutral expressions, whereas men who showed sadness or fear were rated as significantly less dominant than those who showed a neutral expression. As in prior research, men who expressed anger or happiness were rated as more dominant than men who expressed either sadness or fear (see Figure 1).

By contrast, women who showed anger or happiness were perceived as significantly more dominant than those who showed a neutral or fear expression, for which perceptions did not differ. Women who showed a sadness expression were perceived as significantly less dominant than those who showed a neutral expression or fear as well as anger or happiness.

\section{Discussion}

For men, neutral expressions did indeed signal social dominance, and this at levels comparable to the approach emotions of happiness and anger. Also as expected, the two inhibition emotions fear and sadness signaled low level of dominance. Of interest, the pattern for women was different. Whereas the two approach emotions did signal high social dominance and sadness low dominance, the neutral expression was situated in between. That is, for women expressing no emotion did not have the same effect as for men. This latter finding may be explained by the fact that female neutral faces in general are perceived as less dominant than male neutral faces (e.g., Hess et al., 2005) because the facial signals of dominance (such as square jaw, prominent eyebrows, etc.) are more typical for men than for women. Also, participants saw the

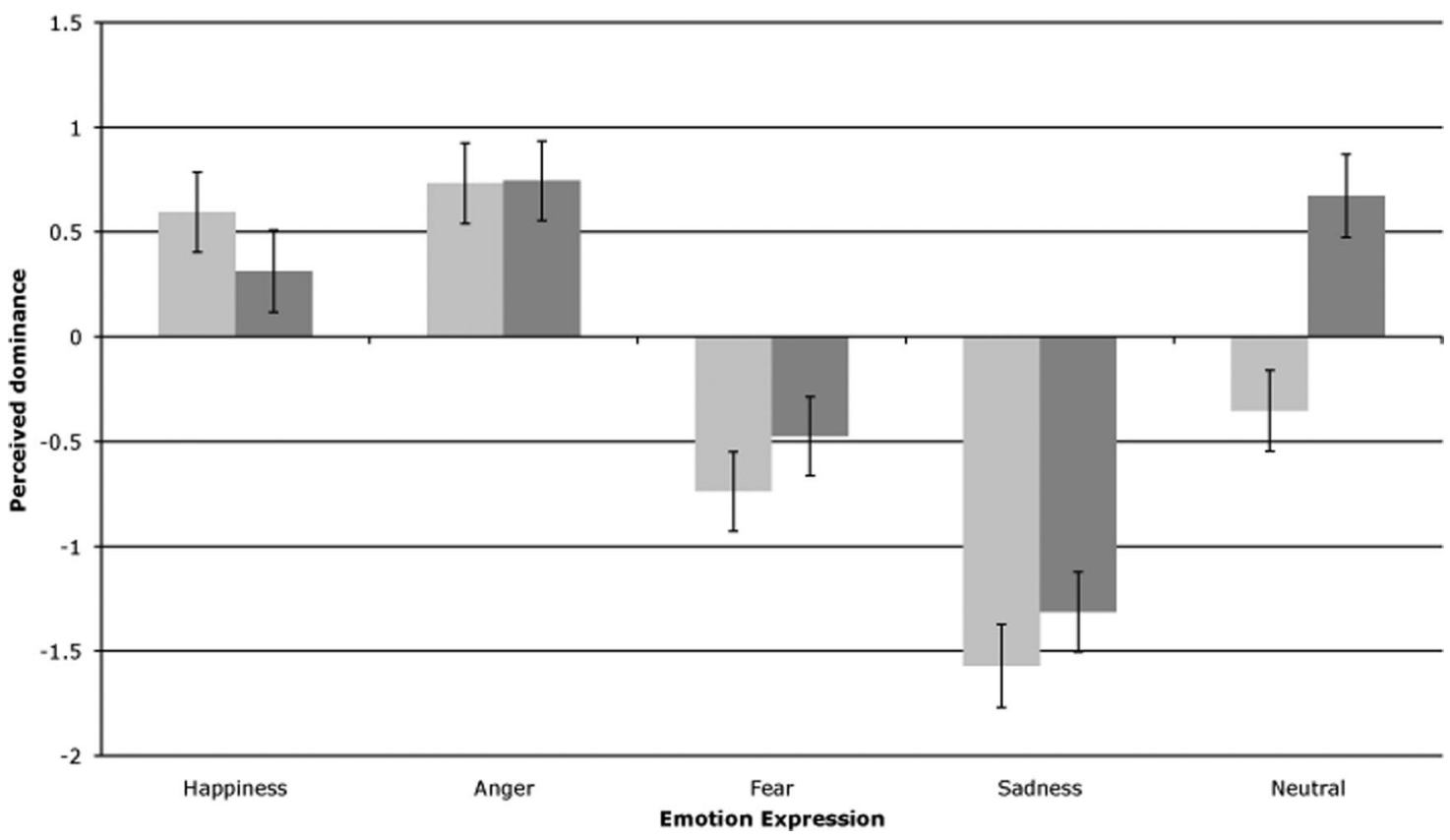

Figure 1. Perceived dominance as a function of encoder gender and emotion. 
men and women only in still photos and no information about the context was provided. Thus, it is possible that the neutral female faces were perceived as disinterested rather than neutral about the situation.

Of interest, the present study showed that, as one would expect based on the model by Keltner et al. (2003), the approach emotion happiness had the same effect on perceived dominance as had anger. This replicates findings by Hess, Bergeron, Cossette, and Hareli (2007) for status conferral. Finally, the present study exemplifies the importance of including a neutral expression as a control condition in experiments with the goal to assess how discrete emotions affect social perceptions as otherwise (a) reactions to male and female faces would have been considered to be more comparable as they were found to be, and (b) differences in perceived power between expressions of emotions such as anger and happiness, on one hand, and sadness and fear, on the other, can be mistakenly attributed to each type of emotion contributing to the perception in question in opposite directions. Apparently, this is not the case when it comes to expressions of men.

Yet, even though facial expressions are an important source for inferences about an individual, they are not the only such source. Most real life encounters occur in stimuli richer environments, which contain a variety of sources of relevant information. That even in such contexts emotion expression remains an important factor was shown by Hess, Adams et al. (2007) who found that narrating an anger event and expressing anger rather than happiness for less than $10 \mathrm{~min}$ out of an almost 2-hr interaction impacted negatively on perceptions of likability.

In Study 2, we therefore showed participants emotional facial expressions, including a neutral expression, in a richer social context. Even though Study 1 suggests the importance of using both male and female stimuli, in this study we used only men. This decision was made because human social contexts are highly norm based and many of these norms are different for men and women. This is especially the case when it comes to the effects of status and nonverbal behavior (Hecht \& LaFrance, 1998; Henley, 1977, 1995; LaFrance, Hecht, \& Paluck, 2003). Thus, to not add confounding elements to this first investigation we chose to restrain the study to male expressers. Also, we decided to assess the impact of emotional expressions on dominance by using two separate and more direct and general measures, one that taps level of dominance and another that taps level of submissiveness. Prior discussions have suggested that submissiveness is not necessarily a "mirror image" of dominance (Moskowitz, 2004). This enabled us to potentially replicate our findings from Study 1 using measures of dominance that are expected to reflect this construct more generally. As mentioned above, the study was conducted in a different culture lower in power distance and comprised a somewhat older sample; both factors are known to be relevant to social power and thus the study allowed for generalization across these factors.

\section{Study 2}

\section{Method}

Participants. A total of 96 individuals (75 men, 19 women, 2 gender unknown) participated. Of these, 79 were managers at different levels, 6 were self-employed, and 10 were nonmanagers. Participants were requested to report their age range, 27 participants were between the ages of 26 and 35, 30 were between 36 and 45, 30 were between 46 and 55, 5 were between 56 and 65,3 above 66 , and 1 participant failed to report age.

Procedure. Participants were recruited via email from a list of over 1,000 people including the pool of alumni of the graduate school of management of the University of Haifa and friends of the authors with work experience. Response rate was around $10 \%$, with no difference in response rate across experimental conditions. List members received one of three different Web links that included the stimulus material and questionnaire for the experiment.

Upon entering the Web site, the participant was asked to watch part of a staged organizational inquiry that was based on an actual failure event. Specifically, a software technician in a company that develops software for electronic commerce is responsible for an upgrade of a customer's system. Following that upgrade, the system crashed and was inoperative for a few hours. The goal of the inquiry was to understand what caused the failure. Participants were told that the short video they would be watching was an excerpt from the entire inquiry in which the employee answers the question "what in your opinion caused the problem?" Each participant watched one of three videos in which the same individual explained that he really does not know what happened. He had followed the relevant instructions and done what he always did in similar situations but this time the system crashed. He further speculated that the fault was a bug in the customer's system. A professional actor played the employee and answered the question in either a neutral emotional tone, or while expressing either anger or shame. The video excerpt was pretested prior to the study to ensure that the desired emotional expression was achieved. The pretest was conducted in a similar population comprised of 71 judges who also received an email with one of three Web links which included the stimulus material followed by three questions tapping the extent to which the object expressed emotional neutrality, anger and shame. Ratings were made on 7-point scales ranging from $0=$ not at all to $6=$ to a large extent. Analyses confirmed that in each condition the desired emotional expression was rated as significantly higher than the other two emotions (results were very similar to the manipulation checks reported below). Raters also converged on these judgments as reflected in the relative high ICCs (for anger, neutrality and shame were $.95, .74$, and .99 , respectively).

After watching the video, participants in the main study rated how dominant and submissive the employee appeared using 7 -point scales anchored with $0=$ not at all and $6=$ to a large extent. A dominant person was described as someone who is the leader type, one that gives orders that are obeyed by others and one who insists on things. A submissive person was described as obedient, accepting orders and obeying them and one who doesn't insist on things. Three 7-point scales anchored with $0=$ not at all and $6=$ to a large extent were used as manipulation checks. Specifically, participants were asked to rate how relaxed, angry, and ashamed the target person was. 


\section{Results}

Manipulation checks. A one-way multivariate analysis of variance (MANOVA) with emotional expression as the independent variable was conducted on relaxed, anger and shame. A significant multivariate main effect emerged for emotional expression, $F(6$, 174) $=36.25, p<.0001, \eta^{2}=.56$. Univariate analyses of variance were conducted as follow-up tests. Significant effects of emotion expression emerged for relaxed, $F(2,89)=54.21, p<$ $.0001, \eta^{2}=.55$, shame, $F(2,89)=24.14, p<.0001, \eta^{2}=.35$, and, anger, $F(2,89)=83.14, p<.0001, \eta^{2}=.65$.

Bonferroni post hoc tests $(p<.05)$ confirmed that the relaxed employee was rated as more relaxed than both the ashamed and angry one respectively $(M=4.32, S D=.95 ; M=3.16, S D=$ $1.39 ; M=1.27, S D=1.08)$. The ashamed employee was also perceived as more relaxed than the angry one. The ashamed employee was rated as experiencing a greater level of shame relative to the relaxed and angry employee respectively $(M=4.00$, $S D=1.65 ; M=2.29, S D=1.51 ; M=1.23, S D=1.55)$. The relaxed employee appeared somewhat more ashamed relative to the angry one. Finally, the angry employee appeared as more angry than the ashamed and relaxed one respectively $(M=5.17, S D=$ $1.05 ; M=1.61, S D=1.48 ; M=1.55, S D=1.18)$. Overall, these analyses confirm that the emotional expressions of the employee were perceived by participants as intended.

Hypotheses. A one-way MANOVA with emotional expression as the independent variable was conducted on dominance and submissiveness. A significant multivariate main effect emerged for emotional expression, $F(4,168)=18.15, p<.0001, \eta^{2}=.30$. Univariate analyses of variance were conducted as follow-up tests. Significant effects of emotion expression emerged for both dominance, $F(2,85)=8.51, p<.0001, \eta^{2}=.17$, and submissiveness, $F(2,85)=38.47, p<.0001, \eta^{2}=.48$.

Bonferroni post hoc tests $(p<.05)$ indicated that the angry employee was rated as more dominant than the one who showed shame. However, the angry employee was not perceived as more dominant than the emotionally neutral employee. Yet, when showing a neutral expression the employee was perceived as only marginally more dominant than when showing shame $(p=.09)$ $(M=1.71, S D=1.35 ; M=3.13, S D=1.34 ; M=2.44, S D=$ 1.22 ; for shame, anger, and emotionally neutral expressions, respectively).

Furthermore, the employee was perceived as about equally submissive when showing either a shame or a neutral expression, but significantly less submissive when showing anger $(M=1.38$, $S D=1.07 ; M=3.73, S D=.98 ; M=3.63, S D=1.26$; for angry, ashamed, and emotionally neutral, respectively). Overall, these findings support the notion that anger signals more dominance than does shame, with neutral being more similar to anger than to shame.

However, as predicted, the difference between anger and shame in perceptions of power was not so much due to the fact that anger increases perceptions of dominance whereas shame reduces them. Rather, shame expressions specifically reduced perceptions of dominance and anger expressions specifically reduced perceptions of submissiveness. The fact that inferences of submissiveness are not a mere mirror reflection of inferences of dominance suggest that to some extent these perceptions are independent from one another (Moskowitz, 2004), especially in more information rich situations that may contain multiple cues for these traits.

Consequently, in the present study, the effect of a neutral expression on perceptions of dominance and submissiveness were also not perfectly parallel. Even though neutral expressions were perceived as dominant as were anger expressions, they were also perceived as submissive as were shame expressions. This may be because in the present context an emotionally neutral expression may also have been perceived as a sign of indifference, thereby confounding the effect of two emotional reactions with different person perception implications.

\section{Discussion}

The goal of the present research was to explore the unique contribution of specific types of emotional expressions by assessing perceptions of dominance for neutral expressions as well. As in previous research, approach-related emotions were associated with higher levels of dominance relative to inhibition-related emotions. Nevertheless, in contrast to what may have seemed to be the conclusions from this prior research (e.g., Tiedens, 2001) and theorizing (Keltner et al., 2003) such differences in perceived dominance are not in all cases attributable to approach-related emotions causing an increase in perceived dominance, whereas inhibition-related emotion causing a decrease. Rather, the present findings show that variations in perceived dominance as a function of expressed emotions vary within different approach and inhibition emotions as well as with gender and further depend on whether dominance or submissiveness is assessed. Also, emotionally neutral expressions seem to convey information that under certain circumstances increases perceptions of dominance.

Specifically, in two studies using different methodologies men's anger (and in Study 1, happiness) led to higher levels of dominance than did the inhibition-related emotions of fear and sadness (Study 1) and shame (Study 2). Nevertheless, dominance perceptions elicited by anger or happiness (Study 1) were not higher than those elicited by a neutral expression.

By contrast, for women, both anger and happiness expressions led to perceptions of higher dominance than was found for neutral expressions. In fact, women with neutral expressions were rated as somewhat submissive, whereas men with neutral expressions were rated as rather dominant. Sadness, however, clearly decreased perceptions of dominance for women as it did for men. Overall, male expressions of approach emotions do not add to perceived dominance over and above an emotionally neutral expression. This may be because men's faces are generally perceived as relatively dominant. By contrast, women gain in perceived dominance when expressing approach-related emotions maybe because they "start off" as lower in dominance than men. In line with this claim, note the difference in Figure 1 between level of dominance of men and women for the neutral expression.

It is noteworthy that submissiveness did not emerge as a mere opposite of dominance. This may in part be attributable to the fact that participants were making both ratings at the same time and hence tried to be subtler in their assessment. Also the situation presented in Study 2 was richer in information and allowed for such subtlety by presenting multiple sources of information. In general, however, it makes sense to assume that not being domi- 
nant does not necessarily mean being submissive and vice versa. This idea is also in line with other social inferences such as arrogance and modesty that despite the fact that they appear to be opposite to one another are not perceived as such (Hareli \& Weiner, 2000).

Overall, the present research explored more specifically how different emotions expressed by an individual contribute to impressions of the individual's dominance or submissiveness. What seems apparent from this research is that approach-related emotions cannot be seen as a set of emotions that indistinguishably affect dominance perceptions. The same is true for inhibition-related emotions. Rather, such effects can be moderated by contextual factors such as the gender of the expresser. What is also clear from the present research is that neutral expressions are perceived by observers as expressions in their own right (Etcoff \& Magee, 1992) and clearly have the potential to signal how a person perceives an emotional event and what kinds of resources the person brings to the situation. Certainly, the results of any such study greatly depend on what one means by an emotionally neutral condition. In fact, as we have suggested, reacting neutrally to a specific emotion-eliciting situation may be perceived either as a sign of indifference or as a sign of competence. Such perceptions may also interact with role expectations. For example, Lewis (2000) found that female managers who reacted neutrally to bad business news were perceived as more competent than were male managerspresumably because of the stereotype of the emotional woman which made the neutral expression shown by a woman appear as reflecting higher level of competence, whereas for a male manager it was simply a role congruent, expected reaction and hence not diagnostic of competence.

The present studies are not without limitations. In both studies, dominance is measured explicitly. This may lead participants to focus on this inference in a way that is likely stronger than it is the case in real-life interactions. Also, in both studies very little information about the target person was provided-in real life situations we often know a person or have second hand information about them. In addition, the fact that participants in Study 2 had to rate at the same time dominance and perceived emotions may have inflated the judged link between the two because it may have made participants more attentive to this link than they may be when they have to judge only one of the two or in real-life situations were judgments are self-generated and may be less explicit. This possibility requires further exploration because it is a shortcoming of most research that studies perception of emotions. Finally, given the different methodology and sample used in each study, it is of importance to test to what extent differences in results between the studies in particular concerning the impact of inhibition-related emotions are because of the different emotions used in each study rather than differences between the studies.

The present research demonstrates the importance of including neutral expressions in studies investigating the influence of emotion expressions on social judgments. In sum, the present research contributes to the emerging literature on the social perception of emotions by zooming in more closely into specific effects of emotions on social judgments related to power.

\section{References}

Aguinis, H., Simonsen, M. M., \& Pierce, C. A. (1998). Effects of nonverbal behavior on perceptions of power bases. The Journal of social psychology, 138, 455-469.

Anderson, C., \& Berdahl, J. L. (2002). The experience of power: Examining the effects of power on approach and inhibition tendencies. Journal of Personality and Social Psychology, 83, 1362-1377.

Aycan, Z., Kanungo, R. N., Mendonca, M., Yu, K., Deller, J., Stahl, G., et al. (2000). Impact of culture on human resource management practices: A 10-country comparison. Applied Psychology: An International Review, 49, 192-221.

Beaupré, M. G., \& Hess, U. (2005). Cross-cultural emotion recognition among Canadian ethnic groups. Journal of Cross-Cultural Psychology, 36, 355-370.

Berger, J., Cohen, B. P., \& Zelditch, Jr., M. (1972). Status characteristics and social interaction. American Sociological Review, 37, 241-255.

Etcoff, N. L., \& Magee, J. J. (1992). Categorial perception of facial expressions. Cognition, 44, 227-240.

Hall, J. A., \& Friedman, G. B. (1999). Status, gender, and nonverbal behavior: A study of structured interactions between employees of a company. Personality and Social Psychology Bulletin, 25, 1082-1091.

Hareli, S., \& Weiner, B. (2000). Accounts for success as determinants of perceived arrogance and modesty. Motivation and Emotion, 17, 215236.

Hecht, M. A., \& LaFrance, M. (1998). License or obligation to smile: The effect of power and sex on amount and type of smiling. Personality and Social Psychology Bulletin, 24, 1332-1342.

Henley, N. M. (1977). Body politics: Power, sex and nonverbal communication. New York: Prentice Hall.

Henley, N. M. (1995). Body politics revisited: What do we know today? In P. J. Kalbfleisch \& M. J. Cody (Eds.), Gender, power, and communication in human relationships (pp. 27-61). Hillsdale, NJ: Erlbaum.

Hess, U., Adams, J., R. B., \& Kleck, R. E. (2005). Who may frown and who should smile? Dominance, affiliation, and the display of happiness and anger. Cognition \& Emotion, 19, 515-536.

Hess, U., Adams, R. B., Jr. \& Kleck, R. E. (2007). Looking at you or looking elsewhere: The influence of head orientation on the signal value of emotional facial expressions. Motivation and Emotion, 31, 137-144.

Hess, U., Bergeron, S., Cossette, M., \& Hareli, S. (2007). Catching flies with honey and vinegar: The influence of smiling and anger displays on perceived status. Submitted for publication.

Hess, U., Blairy, S., \& Kleck, R. E. (2000). The influence of expression intensity, gender, and ethnicity on judgments of dominance and affiliation. Journal of Nonverbal Behavior, 24, 265-283.

Hofstede, G. (2001). Culture's consequences: Comparing values, behaviors, institutions, and organizations across nations (2nd ed.). Thousand Oaks, CA: Sage.

Keltner, D., Gruenfeld, D. H., \& Anderson, C. (2003). Power, approach, and inhibition. Psychological Review, 110, 265-284.

Knutson, B. (1996). Facial expressions of emotion influence interpersonal trait inferences. Journal of Nonverbal Behavior, 20, 165-182.

Kopelman, S., Rosette, A., \& Thompson, L. (2006). The three faces of eve: Strategic displays of positive neutral and negative emotions in negotiations. Organization Behavior and Human Decision Processes, 99, 81101.

LaFrance, M., Hecht, M. A., \& Paluck, E. L. (2003). The contingent smile: A meta-analysis of sex differences in smiling. Psychological Bulletin, 129, 305-334

Levenson, R. W., Ekman, P., \& Friesen, W. V. (1990). Voluntary facial action generates emotion - specific autonomic nervous system activity. Psychophysiology, 27, 363-384.

Lewis, K. M. (2000). When leaders display emotion: How followers respond to negative emotional expression of male and female leaders. Journal of Organizational Behavior, 21, 221-234. 
Matsumoto, D., \& Ekman, P. (1988). Japanese and Caucasian facial expressions of emotion (JACFEE) and neutral faces (JACNeuf) [Slides \& brochure]. San Francisco: San Francisco State University.

Mazur, A. (1985). A biosocial model of status in face-to-face primate groups. Social Forces, 64, 377-402.

Moskowitz, D. S. (2004). Does elevated power lead to approach and reduced power to inhibition? Comment on Keltner, Gruenfeld, and Anderson (2003). Psychological Review, 111, 808-811.

Tiedens, L. Z. (2001). Anger and advancement versus sadness and subjugation: The effect of negative emotion expressions on social status conferral. Journal of personality and social psychology, 80, 86-94.

Tiedens, L. Z., Ellsworth, P. C., \& Mesquita, B. (2000). Stereotypes about sentiments and status: Emotional expectations for high-and low-status group members. Personality and Social Psychology Bulletin, 26, 560-574.

Warner, L. R., \& Shields, S. A. (2007). The perception of crying in women and men: Angry tears, sad tears, and the "right way" to weep (pp. 92-118). In U. Hess \& P. Phillipot (Eds.), Emotion recognition across social groups. Cambridge, MA: Cambridge University Press.

Wilder, C. (1978). From the interactional view: A conversation with Paul Watzlawick. Journal of Communication, 28, 35-45.

Received February 15, 2008

Revision received November 25, 2008

Accepted January 16, 2009 